Review article

Paediatrics Today 2015;11(2):115-125

DOI $10.5457 / \mathrm{p} 2005-114.117$

\title{
NEONATAL THROMBOCYTOPENIA: A COMMON CLINICAL PROBLEM
}

\author{
Jelena ROGANOVIĆ \\ Department of Pediatrics \\ Clinical Hospital Centre \\ Rijeka, Croatia
}

\footnotetext{
*Corresponding author: jelena.roganovic1@ri.t-com.hr Tel.: + 38551659 103/109 Fax.: + 38551623126
}

Received: July 13, 2015

Accepted: September 17, 2015

Key words: Thrombocytopenia Newborn - Platelet transfusion.

\begin{abstract}
The aim of this study was to review current knowledge about neonatal thrombocytopenia. Neonatal thrombocytopenia is a common hematological problem in preterm and sick newborns. The causes of thrombocytopenia are numerous, and the evaluation and the management of the affected neonates may be challenging. The severity of clinical manifestation varies, depending on the platelet count, underlying disease, and bleeding manifestations. The two main underlying pathophysiological mechanisms are increased platelet destruction and decreased platelet production. In many sick neonates, both accelerated platelet destruction and reduced megakaryocytopoiesis may be present simultaneously. Based on the time of onset, neonatal thrombocytopenia can be divided into early, occurring within the first 72 hours, and late, occurring more than 72 hours after birth. This classification is also useful in the diagnostic evaluation. In the majority of cases, thrombocytopenia resolves within a week without intervention. Severe thrombocytopenia is more common in infants admitted to neonatal intensive care units. It may be associated with major bleeding and requires careful management. Platelet transfusions are the mainstay of the treatment, but there are no universally accepted transfusion guidelines in newborns. Conclusion - It is important to identify infants at risk, and if needed, initiate transfusion therapy to prevent severe bleeding and potentially significant morbidity. This review summarizes the current understanding of underlying pathophysiologic mechanisms and management of neonatal thrombocytopenia.
\end{abstract}

\section{Introduction}

Thrombocytopenia is a common hematological abnormality in sick newborns, occurring in up to $25 \%$ of neonates admitted to neonatal intensive care unit (NICU) $(1,2)$. The strongest predictor for neonatal thrombocytopenia is low gestational age. Consequently, thrombocytopenia is a frequent laboratory finding in preterm neonates. Since intrauterine growth restriction is responsible for vast majority of thrombocytopenia cases in preterm newborns, low platelet count might be detected in up to $73 \%$ of extremely low birth weight infants (3).

Neonatal thrombocytopenia is generally defined as a platelet count less than $150,000 / \mu$ l. Degrees of thrombocytopenia can be further subdivided into mild (platelet count 100,000 to $150,000 / \mu \mathrm{l})$, moderate (platelet count 50,000 to $99,000 / \mu \mathrm{l}$ ) and severe (platelet count $<50,000 / \mu \mathrm{l})(4)$. About $75 \%$ of NICU cases are considered mild or moderate, and do not warrant intervention. Among extremely low birth weight infants, 
a high proportion is classified as severe. Severe neonatal thrombocytopenia is associated with significant morbidity, although correlation between platelet count and incidence of bleeding is poor $(3,4)$.

The evaluation and the management of the neonate with thrombocytopenia is a challenge. Therefore, a review of classification, pathogenesis, practical approach, and management of neonatal thrombocytopenia is important.

\section{Classification and pathogenesis}

Neonatal thrombocytopenia can be classified by several different methods. These include: underlying pathologic mechanisms (increased platelet destruction, decreased platelet production, or combination), time of onset (early or late), mode of acquisition (congenital or acquired), gestational age (preterm versus term newborns), and platelet size (small, normal, and large) (4) (Box 1).

This review will focus on classification based on the mechanisms underlying thrombocytopenia in the newborn period. A num-

\begin{tabular}{l} 
Box 1 Classification of Neonatal \\
Thrombocytopenia \\
\hline Degree of thrombocytopenia \\
Mild (platelet count 100,000 to $150,000 / \mu \mathrm{l})$ \\
Moderate (platelet count 50,000 to $99,000 / \mu \mathrm{l})$ \\
Severe (platelet count $<50,000 / \mu \mathrm{l})$ \\
\hline Pathologic mechanisms \\
Increased platelet destruction with sequestration \\
Decreased platelet production \\
Combined mechanisms \\
\hline Time of onset \\
Early ( $<72$ hours of age) \\
Late ( $\geq 72$ hours of age) \\
\hline Mode of acquisition \\
Congenital \\
Acquired \\
\hline Gestational age \\
Preterm newborns \\
Term newborns \\
\hline Platelet size \\
Small \\
Normal \\
Large \\
\hline
\end{tabular}

ber of fetomaternal and neonatal conditions are associated with neonatal thrombocytopenia. Two main mechanisms include accelerated platelet destruction comprising antibodymediated destruction or consumption/sequestration, and reduced platelet production in the bone marrow. In some conditions, a combination of increased platelet destruction and decreased production is responsible for the low platelet count in the newborn.

\section{Increased platelet destruction}

Increased platelet destruction is seen in a number of neonatal conditions. Thrombocytopenia secondary to platelet destruction can be divided into immune and nonimmune. Immune thrombocytopenia is the most common form of accelerated platelet destruction in newborns.

\section{Immune-mediated neonatal thrombocytopenia}

Immune-mediated thrombocytopenia can be subdivided into three mechanisms: transplacental crossing of the maternal antibodies directed against paternally inherited platelet antigen on fetal platelets (termed alloimmune thrombocytopenia), transplacental crossing of cross-reactive maternal autoimmune-derived antiplatelet antibodies (directed against both maternal and fetal platelets), and generation of autoreactive antiplatelet antibodies by the newborn itself (termed autoimmune thrombocytopenia) (5). Distinguishing these conditions by examining the mother's platelet count is crucial, because the severity of these disorders and the management are largely different.

\section{Neonatal alloimmune thrombocytopenia (NAIT)}

NAIT, also referred to as fetomaternal alloimmune trombocytopenia, results from 
transplacental passage of maternal alloantibodies directed against paternal antigens present the fetal platelets, but lacking on maternal platelets. In contrast to $\mathrm{Rh}(\mathrm{D})$ alloimmunization, first-born infants can be also affected. The incidence of NAIT has been estimated at 1 in 2,000 to 3,000 live births (6). The most common alloantigen in white population is human platelet antigen (HPA)1a. The mother is asymptomatic, while clinical findings in affected neonates depend on the severity of thrombocytopenia. Common manifestations include petechiae and bruising in an otherwise well-appearing infant. Intracranial bleeding occurs in $10 \%$ to $15 \%$ of NAIT (4). Laboratory diagnostics includes mother's and father's platelet antigen testing and mother's serum antiplatelet alloantibodies assay. If father's sample is not available, it is useful to perform reactivity of the mother's serum with the newborn's platelets, or to look for the presence of antiplatelet antibodies in the newborn's serum. However, NAIT is primary a clinical diagnosis, and therapy should be initiated in severe cases as soon as the diagnosis is suspected. Term, otherwise healthy infants with no risk factors or with no signs of bleeding are transfused if the platelet count is below $30,000 / \mu \mathrm{l}$ (7). The threshold for transfusion is higher in preterm infants or ill term infants with risk factors. Optimal treatment includes transfusion with platelet concentrates donated by the mother. In cases of severe thrombocytopenia or bleeding, random donor platelets may be used initially. Highdose intravenous immunoglobulin (IVIG), $400 \mathrm{mg} / \mathrm{kg} / \mathrm{day}$ for 3 to 4 consecutive days, or $1 \mathrm{~g} / \mathrm{kg} /$ day for 1 or 2 consecutive days, is effective in prolonging the survival of transfused platelets (8). All infants with NAIT should undergo cranial ultrasonography (4). Because many of intracranial bleedings are of antenatal origin, screening of pregnant women for platelet alloimmunization should be recommended (10).

\section{Immune thrombocytopenia caused by maternal antiplatelet autoantibodies}

This condition occurs in maternal autoimmune disorders, such as immune thrombocytopenia and systemic lupus erythematosus. Passive transplacental transfer of maternal antiplatelet antibodies mediates premature clearance of fetal and newborn platelets. The diagnosis is usually apparent from mother's medical history and low platelet count (11). In this disorder, neonatal platelet counts correlate with the severity of maternal thrombocytopenia, the presence of maternal splenectomy, and an affected older sibling. Thrombocytopenia in the newborn is mild to moderate, and affected infants appear healthy apart from cutaneous bleeding manifestations. In the majority of cases, thrombocytopenia resolves gradually over the first few months of life owing to clearance of maternal antibodies. The risk of intracranial hemorrhage is less than $1 \%(1)$. The treatment depends on the severity of thrombocytopenia, clinical findings, and the risk of bleeding. Platelet transfusion is given only to infants with major haemorrhage. Transfusions are not effective because antibodies react with all donor platelets, including maternal. IVIG (1 $\mathrm{g} / \mathrm{kg}$ ) should be administered to infants with severe thrombocytopenia, and typically produces a rapid response. In persistent cases, a short course of prednisone $(2 \mathrm{mg} / \mathrm{kg} /$ day $)$ or methylprednisolone $(1 \mathrm{mg} / \mathrm{kg} /$ twice a day for 5 days) may be given, but there is no clear utility of this approach $(5,12)$.

\section{Autoimmune thrombocytopenia}

True autoimmune thrombocytopenia, in which neonates generate antibodies against their own platelets, is very uncommon in the newborn period. It commonly indicates an underlying immune disorder (5). 


\section{Drug-related thrombocytopenia}

Several drugs have been reported to cause thrombocytopenia. If drug-induced maternal thrombocytopenia is mediated by immunoglobulin $\mathrm{G}(\mathrm{IgG})$ antibodies, they can cross the placenta and destroy fetal platelets. Implicated medications include penicillin, digoxin, antiepileptic drugs, quinidine, and indomethacin. Drug-related thrombocytopenia can also be caused by bone marrow suppression (4).

\section{Nonimmune thrombocytopenia}

Nonimmune neonatal thrombocytopenia as a result of increased platelet destruction is seen in a number of conditions. These include bacterial sepsis, viral infections (rubella virus, herpes simplex virus, cytomegalovirus, human herpesvirus 6 , echovirus, human immunodeficiency virus), protozoal infections (Toxoplasma gondii), disseminated intravascular coagulation (DIC), vascular anomalies, thromboembolic complications, polycythemia, exchange transfusion, and rare others. In premature infants, thrombocytopenia often complicates respiratory distress syndrome, persistent pulmonary hypertension, necrotizing enterocolitis, and hyperbilirubinemia treated by phototherapy $(4,5)$.

\section{Sepsis}

Bacterial sepsis and necrotizing enterocolitis are common causes of nonimmune thrombocytopenia in NICU (13). Multiple mechanisms are responsible for sepsis-induced thrombocytopenia, and include endothelial damage, accelerated platelet destruction and their removal by the reticuloendothelial system, consumption resulting from DIC, platelet aggregation due to binding of bacterial products to platelet membranes, and decreased production. Thrombocytopenia is generally considered as the first sign of neonatal septicemia $(14,15)$. The average duration of bacterial sepsis-induced thrombocytopenia is 6 days (3)

\section{Disseminated intravascular coagulation}

DIC is a systemic process that comprises both hemorrhage and thrombosis. DIC is a complication of an underlying illness, such as sepsis, necrotizing enterocolitis, asphyxia, meconium aspiration, or severe respiratory distress syndrome. The affected newborn is ill-appearing, thrombocytopenia is moderate to severe, and microangiopathic changes are present on the peripheral blood smear (16). Confirming laboratory findings include prolonged prothrombin time and activated partial thromboplastin time, decreased fibrinogen, and increased fibrin degradation product or D-dimer levels. Management is directed to the underlying disorder, altogether with platelet transfusions and fresh frozen plasma (4).

\section{Thrombosis and polycythemia}

Thrombosis causes neonatal thrombocytopenia by increased platelet consumption. Patients in NICU are at risk for thrombosis due to the use of indwelling vascular catheters and the presence of serious medical conditions or events. Patients should be evaluated for thromboembolic disorders if thrombocytopenia cannot be related to other conditions. Another cause of thrombocytopenia in near-term or term neonates is polycythemia. Polycythemia is thought to be due to increased platelet aggregation and activation, and decreased megakaryopoiesis due to increased erythropoietin production (17).

\section{Vascular anomalies}

Certain types of vascular anomalies are associated with thrombocytopenia. Kasabach- 
Merritt syndrome consists of rapidly growing hemangioendothelioma, thrombocytopenia, and coagulopathy. Thrombocytopenia results from shortened platelet survival caused by platelet sequestration in the vascular malformation, and approximately half of the patients experience systemic bleeding during the first months of life $(4,5)$. Management includes measures for vascular lesion regression and transfusion therapy. Any infant with unexplained thrombocytopenia, with or without evidence of DIC, should be evaluated for hidden vascular lesions, especially of the liver or spleen.

\section{Decreased platelet production}

Thrombocytopenia secondary to decreased platelet production is rare, accounting for less than $5 \%$ of thrombocytopenic newborns (5). Causes include genetic disorders and diseases associated with bone marrow infiltration or suppression. Genetic disorders can result in thrombocytopenia combined with other clinical findings or in isolated thrombocytopenia.

\section{Thrombocytopenia-absent radius syndrome}

Thrombocytopenia-absent radius syndrome is an autosomal recessive disorder characterized by severe thrombocytopenia at birth or during the first week of life and bilateral radial aplasia. Other anomalies of the skeleton (upper and lower limbs, ribs, and vertebrae), heart (septal defects, tetralogy of Fallot), genitourinary and gastrointestinal system can occur. Low platelet count is due to hypo/ dysmegakaryopoiesis, and other hematopoietic lineages are not affected. Treatment consists of platelet transfusions when needed. By several months of age, increased number of megakaryocytes usually appears, with subsequent rise in platelet counts. Hematopoietic cell transplantation is an option for patients who remain thrombocytopenic with bleeding $(5,18)$.

\section{Congenital amegakaryocytic thrombocytopenia}

Congenital amegakaryocytic thrombocytopenia is a rare disorder characterized by severe thrombocytopenia in the newborn period and near absence of megakaryocytes in the bone marrow. Patients are at high risk for the development of complete bone marrow failure within the first few years of life. Bone marrow transplantation is the only effective treatment (19).

\section{Fanconi anemia}

Fanconi anemia is an autosomal recessive disorder characterized by various physical anomalies (short stature, skin, skeletal, craniofacial and genitourinary malformations) and pancytopenia. The presentation in the newborn period is exceedingly rare. Transfusions, androgen therapy and hematopoietic growth factors can help bone marrow failure temporarily, but the long-term treatment is bone marrow transplantation if a donor is available (1).

\section{Wiskott-Aldrich syndrome}

Wiskott-Aldrich syndrome is an X-linked recessive disorder that presents with immunodeficiency, eczema, and thrombocytopenia with small platelets. Male infants usually present with prolonged bleeding after circumcision, purpura, bloody diarrhea, or unusual bruising. Patients with thrombocytopenia may require IVIG and/or corticosteroids. Platelet and/or red blood cell transfusions are indicated in severe bleeding. Bone marrow transplantation may be curative, with correction of hematologic and immunologic defects and resolving of eczema $(20,21)$. 


\section{May-Hegglin anomaly}

May-Hegglin anomaly, also known as Dohle leukocyte inclusions with giant platelets and macrothrombocytopenia with leukocyte inclusions, is an autosomal dominant disorder characterized by various degrees of thrombocytopenia. Peripheral blood smear reveals giant platelets, and leukocyte inclusion bodies (22).

\section{Bernard-Soulier syndrome}

Bernard-Soulier syndrome, also called hemorrhagiparous thrombocytic dystrophy, is an autosomal recessive disorder presenting with mild thrombocytopenia, giant platelets, and bleeding (21).

\section{Bone marrow suppression and bone marrow infltration}

Congenital viral infections with rubella virus, cytomegalovirus, herpesvirus, human herpesvirus 6 and human immunodeficiency virus, have been associated with neonatal thrombocytopenia, mainly because of damaged megakaryopoiesis and thrombopoiesis. Transplacental passage of drugs or the use of drugs in neonates can cause thrombocytopenia as a consequence of bone marrow suppression, besides the development of drug-dependent antibodies. Drugs implicated are salicylates, quinine, hydralazine, tolbutamide, and thiazide $(5,23)$. Chronic fetal hypoxia with placental dysfunction and intrauterine growth restriction in preterm infants, and perinatal asphyxia with acute hypoxia, are both associated with decrease in platelet production (11). Birth asphyxia is also associated with DIC, which may cause platelet activation and consumption.

Neonatal malignant diseases characterized by bone marrow infiltration, such as congenital leukemia and metastatic neuroblastoma, can be accompanied by thrombocytopenia. Other hematopoietic lineages are typically affected. Up to $10 \%$ of infants with Down syndrome are at risk to develop transient myeloproliferative disorder restricted to the neonatal period. This megakaryoblastic proliferative disease is characterized by leukocytosis with the presence of peripheral blasts, thrombocytopenia, anemia, and hepatic involvement. Most affected children will have a spontaneous resolution of their disease over the first few months of life, and most will not ultimately develop acute myeloid leukemia (24).

\section{Onset of thrombocytopenia}

Depending on the time of onset, neonatal thrombocytopenia can be subdivided into two major groups. Early-onset thrombocytopenia occurs within 72 hours after birth, and late-onset thrombocytopenia occurs more than 72 hours after birth.

\section{Early-onset thrombocytopenia}

In majority of preterm neonates, early thrombocytopenia is frequently associated with fetomaternal conditions, such as placental insufficiency with intrauterine growth restriction, and complicated pregnancies (preeclampsia, pregnancy-induced hypertension, maternal diabetes). It is considered that chronic fetal hypoxia induces erythropoiesis, which suppresses megakaryopoiesis and platelet production. In these cases, thrombocytopenia is usually mild to moderate, resolves within 10 days, and requires no treatment (11). By contrast, severe early thrombocytopenia is much less common and occurs in term as well as preterm neonates. The usual causes are severe perinatal infections (Escherichia coli, group B streptococcus) and perinatal asphyxia, both often with DIC. In term neonates, antibodymediated platelet destruction is the most important cause of early-onset thrombocytopenia (NAIT and immune thrombocytopenia 
caused by maternal antiplatelet autoantibodies) $(25,26)$.

\section{Late-onset thrombocytopenia}

Late-onset thrombocytopenia is most frequently related to acquired bacterial infections, such as sepsis and necrotizing enterocolitis. It is usually profound and progresses rapidly, and may take several weeks to recover. DIC is often a significant contributor. Affected newborns require intensive care and multiple platelet transfusions (4). Other causes of late thrombocytopenia include viral infections (herpes simplex virus, cytomegalovirus), catheter-related thrombosis, and drug-induced thrombocytopenia (27).

\section{Diagnostic evaluation}

When assessing thrombocytopenia in a newborn, several key questions should be addressed. What is the degree of thrombocytopenia? Is the patient term or preterm infant? Is thrombocytopenia of early- or late-onset? Is the child ill- or healthy-appearing? What are the bleeding manifestations? Are other comorbidities present? Are there any findings suggestive of a congenital infection? Are there any congenital anomalies or dysmorphic features?

In cases of severe thrombocytopenia and symptomatic patients, any diagnostic evaluation is set aside by prompt initial management. The evaluation is directed on determining the underlying cause and establishing the specific therapy. The diagnostic approach is focused on maternal and neonatal history, and the infant's clinical condition (28).

\section{History}

The following information from the history can help guide the diagnostic evaluation. Maternal conditions associated with neonatal thrombocytopenia are immune throm- bocytopenia, systemic lupus erythematosus, cytomegalovirus infection, or drug exposure. Previously affected child in the same family might be suggestive of NAIT. Abnormal fetal monitoring or neonatal resuscitation after delivery may be indicative of perinatal asphyxia. Preterm newborns are more likely to have thrombocytopenia caused by placental insufficiency, congenital infections, perinatal asphyxia, or perinatal infections. In term newborns, the most common is immune-mediated thrombocytopenia. Early-onset thrombocytopenia is more often related to complications of pregnancy (placental insufficiency) or delivery (perinatal asphyxia), or from maternal-antibody platelet destruction (NAIT). On the contrary, late-onset thrombocytopenia is usually due to acquired systemic condition (sepsis, necrotizing enterocolitis).

\section{Clinical setting}

Well-appearing neonates are more likely to have immune-mediated or genetic causes of thrombocytopenia. In contrast, in ill-appearing patients thrombocytopenia is more often associated with systemic disease (sepsis with or without DIC, necrotizing enterocolitis, perinatal asphyxia). Dysmorphic features and specific physical findings might be indicative for several syndromes (thrombocytopenia-absent radius syndrome, Fanconi anemia, chromosomal disorders due to trisomy 13,18 , or 21 , and Turner syndrome). Jaundice or hepatosplenomegaly may suggest congenital infection $(29,30)$.

\section{Laboratory studies}

The complete blood count usually shows isolated thrombocytopenia. Associated anemia and/or neutropenia suggest bone marrow suppression. It should be considered that $5 \%$ to $10 \%$ of thrombocytopenias are due to laboratory errors, and therefore, in the absence of bleeding signs, platelet count 
measurement should always be repeated (11). Peripheral blood smear examination, along with mean platelet volume and reticulated platelet count, reveals the platelet morphology and size, which are helpful in distinguishing decrease in platelet production (normal or small-size platelets) and increase in platelet destruction (large platelets), and detection of congenital platelet disorders. In the newborn with mild to moderate thrombocytopenia, whose platelet count improves, no further diagnostic testing is indicated. Other laboratory tests are performed according to the clinical setting. Further testing should never delay platelet transfusion if required. Ill-appearing neonates, who are more likely to have DIC and sepsis, require coagulation studies (prothrombin time, activated partial thromboplastin time, fibrinogen, d-dimer), and blood cultures. Mother's platelet count should be checked (31). Evaluation for NAIT includes platelet antigen typing of the mother, father, and newborn, and testing the mother's serum for antiplatelet alloantibodies. If available, DNA testing is performed to identify platelet antigen genotypes (11). Bone marrow aspiration is rarely needed as a direct diagnostic tool to determine the underlying pathophysiologic mechanism. Impaired megakaryopoiesis confirms low platelet production, while accelerated megakarypoiesis signifies increased platelet production. The length of time between transfusions indicates the velocity of platelet destruction.

\section{Differential diagnosis}

The differential diagnosis includes inherited clotting disorders and qualitative platelet abnormalities. Disorders of blood coagulation have normal platelet count and abnormal coagulation studies. In the type $2 \mathrm{~B}$ von Willebrand disease, increased affinity of abnormal von Willebrand factor for its receptor on the platelet may lead to platelet ag- gregation and thrombocytopenia. Disorders of platelet function are inherited or acquired. Some inherited platelet disorders have a normal platelet count, and others present with thrombocytopenia. Acquired impairment of platelet function may occur due to pathologic conditions in either mother or infant, or use of certain drugs. Causative maternal factors include diabetes, dietary abnormalities, smoking, ethanol abuse, and use of drugs (salicylate). In infants, contributory conditions are perinatal aspiration syndrome, renal failure, hepatic failure, drugs (indomethacin, ibuprofen, ampicillin, and inhaled nitric oxide), hyperbilirubinemia, and phototherapy. When platelets are exposed to blue fluorescent light in vitro, transient thrombocytopenia with decreased platelet aggregation and morphological platelet changes occur (28).

\section{Management}

In the majority of affected newborns, thrombocytopenia resolves spontaneously within a week and requires no intervention. Specific therapy should be given to patients with identified etiology. Treatment of neonatal thrombocytopenia is based on administration of platelet transfusions. There are no universally accepted guidelines for platelet transfusions in newborns. Due to lack of evidence, current guidelines differ widely not only between countries, but also between hospitals in the same country. Platelet transfusions can be given to treat active bleeding (therapeutic transfusions), or to prevent bleeding (prophylactic transfusions) (11). Up to $98 \%$ of platelet transfusions in neonates are given prophylactically (32).

\section{Indications}

The platelet level at which to transfuse in order to prevent bleeding is uncertain, with platelet counts varying from 20,000 to $100,000 / \mu \mathrm{l}(28)$. As a rule, platelet transfu- 
sions should be given to the neonate when the degree of thrombocytopenia, alone or in combination with other factors, results in an unacceptable risk of hemorrhage. However, risk of hemorrhage is often difficult to assess. Most experts recommend to determine the threshold for platelet transfusion by the clinical setting. For stable term newborn, platelet threshold is $20,000 / \mu \mathrm{l}$. In preterm and unstable infants, transfusion is considered if the platelet count is $<50,000 / \mu \mathrm{l}$; some institutions apply a more restrictive threshold of $<30,000 / \mu \mathrm{l}$ in this setting. Newborns with serious concurrent conditions (sepsis, DIC), those who are receiving drugs that may impair platelet function or who are being treated with anticoagulants, should be transfused at higher platelet levels (i.e. $<50,000 / \mu \mathrm{l}$ ). Platelet transfusion is recommended for any neonate with active bleeding and a platelet count $<100,000 / \mu \mathrm{l}(33,34)$. For neonates who are treated by extracorporeal membrane oxygenation and those requiring major surgery, most centers advocate keeping the platelet count $>100.000 / \mu \mathrm{l}(28)$.

\section{Administration}

The recommended dose for platelet transfusion in neonates is 10 to $15 \mathrm{ml} / \mathrm{kg}$, which produces the increase in platelet count from 50,000 to $100,000 / \mu \mathrm{l}$. Volume reduction should not be done unless there is a need for strict fluid restriction (heart failure, renal failure, generalized edema). Volume reduction can cause platelet activation due to centrifugation. The activation of platelets may affect platelet metabolism and platelet function, and cause febrile non-hemolytic transfusion reactions due to the release of cytokines and other factors (35). In most cases, platelets from a random donor can be used (36). Platelets should be ABO-matched and leukocytes removed, thereby providing cytomegalovirus-safe product (11). Irradiation is rec- ommended for certain subgroups of patients to prevent transfusion-related graft-versushost disease: premature infants, infants with known immunodeficiency, and all neonates for whom platelets are donated by a relative. Bacterial contamination is the most common infectious risk of platelet transfusion, due to the storage at room temperature. It occurs in approximately 1 of 2,000 to 3,000 platelet transfusions (37). The transfusion should be administered as soon as it arrives on the ward. It is usually given through a peripheral intravenous catheter. Platelet transfusion is given as rapidly as the newborn will tolerate the volume of suspension; this is usually over 30 minutes and no longer than two hours. Postransfusional monitoring of the platelet count is performed in the time intervals depending of the gestational age, presumed etiology, severity of the condition, and the response to prior transfusions. NAIT and immune thrombocytopenia caused by maternal antiplatelet autoantibodies require a specific therapeutic approach (as discussed earlier) and monitoring. In NAIT, platelet counts need to be monitored until normalization and at least until 5 days after birth. In infants from mothers with immune thrombocytopenia, lower platelet count can last up to 3 months. Clinical endpoints, i.e. bleeding, are the most important method for evaluating efficacy of platelet transfusions.

Different scores have been developed to improve objective assessment of bleeding, but lack of standardization of these methods is still a major problem. Studies on alternatives to platelet transfusions, addressing the use of growth factors, are under investigation. Until then, an individualized patient management is the safest and most efficient approach $(38,39)$.

\section{Conclusion}

Neonatal thrombocytopenia is a common clinical entity in NICU. The diagnostic 
evaluation is focused on defining underlying pathophysiological mechanisms and, if possible, directing specific therapy. The right diagnosis can be difficult when multiple comorbidities are present. In infants with severe thrombocytopenia or in symptomatic infants with active bleeding, initial management has the advantage over any diagnostic evaluation. Platelet transfusions are the hallmark of the management of neonatal thrombocytopenia. However, evidence regarding target platelet level is lacking. Harmonized guidelines are necessary to standardize treatment and support clinical management decisions.

Aknowledgments: I am grateful to dr Izabela Kranjčec, Children's Hospital Zagreb, for helping me with preparation of the manuscript.

Conflict of interest: The author declares that she has no conflict of interest.

\section{References}

1. Roberts I, Murray NA. Neonatal thrombocytopenia: causes and management. Arch Dis Child Fetal Neonatal Ed. 2003;88(5):F359-64.

2. Stanworth SJ. Thrombocytopenia, bleeding, and use of platelet transfusions in sick neonates. Hematology Am Soc Hematol Educ Program. 2012:5126.

3. Medscape Multispecialty [homepage on the Internet]. Neonatal Thrombocytopenia: Evaluation and Management. Medscape Nurses LLC; 2007 [cited 2015 Jul 2]. Available from: http://www.medscape. org/viewarticle/553177

4. UpToDate [homepage on the Internet]. Causes of neonatal thrombocytopenia. [updated $2015 \mathrm{Mar}$ 26; cited 2015 Jul 4]. Available from: http://www. uptodate.com/contents/causes-of-neonatal-thrombocytopenia

5. Cantor AB. Hemostasis in the Newborn and Infant. In: Orkin SH, Fisher DE, Look AT, Lux SE, Ginsburg D, Nathan DG, editors. Nathan and Oski's Hematology and Oncology of Infancy and Childhood. 8th ed. Philadelphia, PA: Elsevier Saunders; 2015. p. 128-50.

6. Roganovic J, Kranjcec I. Neonatal alloimmune thrombocytopenia. Int J Pediat Health Care Adv. 2(1): 4-6.
7. Bassler D, Greinacher A, Okascharoen C, Klenner A, Ditomasso J, Kiefel V, et al. A systematic review and survey of the management of unexpected neonatal alloimmune thrombocytopenia. Transfusion. 2008;48(1):92-8.

8. Ouwehand WH, Smith G, Ranasinghe E. Management of severe alloimmune thrombocytopenia in the newborn. Arch Dis Child Fetal Neonatal Ed. 2000;82(3):F173-5.

9. Bussel J. Diagnosis and management of the fetus and neonate with alloimmune thrombocytopenia. J Thromb Haemost. 2009;7(Suppl 1):S253-7.

10. Kamphuis MM, Paridaans NP, Porcelijn L, Lopriore E, Oepkes D. Incidence and consequences of neonatal alloimmune thrombocytopenia: a systematic review. Pediatrics. 2014;133(4):715-21.

11. Gunnink SF, Vlug R, Fijnvandraat K, van der Bom JG, Stanworth SJ, Lopriore E. Neonatal thrombocytopenia: etiology, management and outcome. Expert Rev Hematol. 2014;7(3):387-95.

12. Van der Lugt NM, van Kampen A, Walther FJ, Brand A, Lopriore E. Outcome and management in neonatal thrombocytopenia due to maternal idiopathic thrombocytopenic purpura. Vox Sang. 2013;105(3):236-43.

13. Sola-Visner M, Sallmon H, Brown R. New insights into the mechanisms of non-immune thrombocytopenia in the neonate. Semin Perinatol. 2009;33(1):43-51.

14. Arif SH, Ahmad I, Ali SM, Khan HM. Thrombocytopenia and bacterial sepsis in neonates. Indian J Hematol Blood Transfus. 2012;28(3):147-51.

15. Baer VL, Lambert DK, Henry E, Christensen RD. Severe thrombocytopenia in the NICU. Pediatrics. 2009;124(6):e1095-100.

16. Sola MC, Del Vecchio A, Rimsza LM. Evaluation and treatment of thrombocytopenia in the neonatal intensive care unit. Clin Perinatol. 2000;27(3):655-79.

17. Acunas B, Celtik C, Vatansever U, Karasalihoglu S. Thrombocytopenia: an important indicator for the application of partial exchange transfusion in polycythemic newborn infants? Pediatr Int. 2000;42(4):343-7.

18. Toriello HV. Thrombocytopenia-absent radius syndrome. Semin Thromb Hemost. 2011;37(6):70712.

19. Ghauri RI, Naveed M, Mannan J. Congenital amegakaryocytic thrombocytopenic pur- 
pura (CAMT). J Coll Physicians Surg Pak. 2014;24(4):285-7.

20. Balduini CL, Pecci A, Noris P. Diagnosis and management of inherited thrombocytopenias. Semin Thromb Hemost. 2013;39(2):161-71.

21. Ariga T. Wiskott-Aldrich syndrome; an $\mathrm{x}$ linked primary immunodeficiency disease with unique and characteristic features. Allergol Int. 2012;61(2):183-9.

22. Balduini CL, Pecci A, Savoia A. Recent advances in the understanding and management of MYH9related inherited thrombocytopenias. Br J Haematol. 2011;154(2):161-74.

23. Fernández KS, Alarcón P. Neonatal thrombocytopenia. NeoReviews 2014;14(2):e74-e82.

24. Gamis AS, Smith FO. Transient myeloproliferative disorder in children with Down syndrome: clarity to this enigmatic disorder. Br J Haematol. 2012;159(3):277-87.

25. Bolat F, Kılıç SÇ, Oflaz MB, Gülhan E, Kaya A, Güven AS, et al. The prevalence and outcomes of thrombocytopenia in a neonatal intensive care unit: a three-year report. Pediatr Hematol Oncol. 2012;29(8):710-20.

26. Christensen RD, Henry E, Del Vecchio A. Thrombocytosis and thrombocytopenia in the NICU: incidence, mechanisms and treatments. J Matern Fetal Neonatal Med. 2012;25(Suppl 4):S15-7.

27. Del Vecchio A. Evaluation and management of thrombocytopenic neonates in the intensive care unit. Early Hum Dev. 2014;90(Suppl 2):S51-5.

28. UpToDate [homepage on the Internet]. Clinical manifestations, evaluation, and management of neonatal thrombocytopenia. [updated 2015 Mar 26; cited $2015 \mathrm{Jul} 4$ 4]. Available from: http://www. uptodate.com/contents/clinical-manifestationsevaluation-and-management-of-neonatal-thrombocytopenia

29. Bussel JB, Sola-Visner M. Current approaches to the evaluation and management of the fetus and neonate with immune thrombocytopenia. Semin Perinatol. 2009;33(1):35-42.

30. Wong W, Glader B. NeoReviews. 2004;5(10):e44450.

31. Chakravorty S, Roberts I. How I manage neonatal thrombocytopenia. $\mathrm{Br} \mathrm{J}$ Haematol. 2012;156(2):155-62.

32. Stanworth SJ, Clarke P, Watts T, Ballard S, Choo L, Morris T, et al. Prospective, observational study of outcomes in neonates with severe thrombocytopenia. Pediatrics. 2009;124(5):e826-34.

33. Josephson CD, Su LL, Christensen RD, Hillyer $\mathrm{CD}$, Castillejo MI, Emory MR, et al. Platelet transfusion practices among neonatologists in the United States and Canada: results of a Survey. Pediatrics. 2009;123(1):278-85.

34. Cremer M, Sola-Visner M, Roll S, Josephson CD, Yilmaz Z, Bührer C, Dame C. Platelet transfusions in neonates: practices in the United States vary significantly from those in Austria, Germany, and Switzerland. Transfusion. 2011;51(12):2634-41.

35. Gulliksson H. Platelets from platelet-rich-plasma versus buffy-coat-derived platelets: what is the difference? Rev Bras Hematol Hemoter. 2012;34(2):76-7.

36. Kiefel V, Bassler D, Kroll H, Paes B, Giers G, Ditomasso J, et al. Antigen-positive platelet transfusion in neonatal alloimmune thrombocytopenia (NAIT). Blood. 2006;107(9):3761-3.

37. Corash L. Bacterial contamination of platelet components: potential solutions to prevent transfusionrelated sepsis. Expert Rev Hematol. 2011;4(5):50925.

38. Holbro A, Infantia L, Sigleb J, Busera A. Platelet transfusion: basic aspects. Swiss Med Wkly. 2013;143:w13885.

39. Christensen RD, Carroll PD, Josephson CD. Evidence-based advances in transfusion practice in neonatal intensive care units. Neonatology. 2014;106(3):245-53. 